\title{
Análisis de la biocompatibilidad de Ti6Al4V y acero inoxidable 316 LVM basado en efectos de pH, aplicando criterios de la norma ASTM F2129
}

\author{
Analysis of the biocompatibility of Ti6Al4V and stainless steel 316 LVM \\ based on effects of pH, applying criteria of ASTM F2129 standard \\ Jeniffer Caballero Sarmiento $^{1 *} \quad$ Estefanía Correa Muñoz ${ }^{1} \quad$ Hugo Estupiñan Duran $^{1}$ \\ Recibido 7 de enero de 2016, aceptado 29 de junio de 2016 \\ Received: January 7, 2016 Accepted: June 29, 2016
}

\begin{abstract}
RESUMEN
Se estudió el efecto del pH en el comportamiento de la corrosión del acero inoxidable 316 LVM y la aleación de Ti6Al4V ELI mediante técnicas electroquímicas, como Polarización Potenciodinámica Cíclica y Espectroscopia de Impedancia Electroquímica. La velocidad de corrosión se calculó por extrapolación de las pendientes Tafel y se analizó la susceptibilidad a las picaduras de cada uno de los materiales sometidos a condiciones de concentración de $3 \%$ de $\mathrm{NaCl}$ y un amplio rango de $\mathrm{pH}$ (4,5-67,5-10-12). Los resultados obtenidos indicaron que las condiciones de $\mathrm{pH} 4,5$ tienen un efecto negativo en la velocidad de corrosión de ambos materiales comparado con los otros $\mathrm{pH}$.
\end{abstract}

Palabras clave: Velocidad de corrosión, susceptibilidad a la picadura, impedancia electroquímica, polarización potenciodinámica cíclica, biomateriales.

\section{ABSTRACT}

The effect of $\mathrm{pH}$ on corrosion behaviour in stainless steel $316 \mathrm{LVM}$ and alloy Ti6Al4V was studied by various electrochemical techniques such as cyclic potentiodynamic polarization and electrochemical impedance spectroscopy (EIS). The corrosion rate was calculated with Tafel extrapolations and the pitting corrosion was also analysed for each material in different conditions of concentration $3 \%$ of $\mathrm{NaCl}$ and range $\mathrm{pH}$ (4.5-6-7.5-10-12). The results exhibited that the conditions of $\mathrm{pH} 4,5$ has negative effect in the corrosion rate for both materials compared with other $\mathrm{pH}$.

Keywords: Corrosion rate, pitting corrosion, electrochemical impedance, cyclic potentiodynamic polarization, biomaterials.

\section{INTRODUCCIÓN}

La propiedad más importante que debe tener un material para uso biomédico es la biocompatibilidad seguida por la resistencia a la corrosión. Los principales materiales metálicos empleados son aceros inoxidables, aleaciones de cobalto, titanio y aleaciones de titanio. Los aceros inoxidables fueron los primeros materiales en ser usados como biomateriales y el titanio-aleaciones de titanio son nuevos materiales en este campo que poseen ventajas tan importantes como mejor biocompatibilidad y excelentes propiedades mecánicas [1].

\footnotetext{
1 Departamento de Materiales y Minerales. Universidad Nacional de Colombia. Cl 75 \#79 a-51. Medellín, Colombia. E-mail: jscaballeros@unal.edu.co; ecorream@unal.edu.co; haestupinand@unal.edu.co

* Autor de correspondencia
} 
El Ti6Al4V es la aleación de titanio más usada en implantes, pero estudios indican que para aplicaciones en implantes permanentes es posible que tenga efectos tóxicos debido a la liberación de vanadio y aluminio. Por tal razón se han desarrollado investigaciones en las principales propiedades responsables de la biocompatibilidad, como: bajo nivel de conductividad electrónica, alta resistencia a la corrosión, estado termodinámico a condiciones de $\mathrm{pH}$ en medio fisiológico, entre otras [1-2].

Por su parte, los aceros inoxidables austeníticos como el acero 316 LVM se utilizan y recomiendan en implantes temporales (placas, tornillos y clavos), debido a que se conoce que su resistencia a la corrosión es baja comparada con otros materiales [3].

Todos estos materiales antes de ser usados en implantes, deben ser evaluados mediante procesos donde son expuestos a un medio fisiológico simulado. Teniendo en cuenta que se trata de un medio electrolítico que contiene iones cloruro con un $\mathrm{pH}$ 7,4 aproximadamente que puede variar según las condiciones del cuerpo humano, así cuando se presentan infecciones el $\mathrm{pH}$ es alcalino, en presencia de heridas y hematomas el $\mathrm{pH}$ es de 5,2 y 4 respectivamente [4]. La norma ASTM F2129, adaptada de las normas ASTM G5 y G61, permite evaluar la resistencia de dispositivos metálicos implantables a la picadura y la corrosión localizada en ambientes fisiológicos y su mayor importancia se da por la posibilidad de reemplazar ensayos clínicos por criterios válidos del análisis del ensayo dado por la norma [5-6].

La investigación en el comportamiento de la velocidad de corrosión en las aleaciones de titanio ha tomado bastante importancia en los últimos años. Sin embargo, hay pocos estudios que evalúen dicho comportamiento mediante técnicas electroquímicas en las aleaciones sin un tratamiento previo, es decir, se evalúa esto en aleaciones con recubrimientos. De acuerdo a la literatura, la velocidad de formación y las características de la película pasiva determinan la resistencia a la corrosión de la aleación, las que dependen de las condiciones a las que son sometidas las aleaciones (temperatura, concentración, pH) [7].

Cvijović y colaboradores investigaron el comportamiento electroquímico de las aleaciones Ti13Nb13Zr y Ti6Al4V mediante Espectroscopia de Impedancia Electroquímica e indicaron que en esta última aleación se formó una película pasiva más estable y, por ende, presenta una alta resistencia a la corrosión [8].

Wen y colaboradores, estudiaron el comportamiento de la aleación de Ti6A14V mediante técnicas electroquímicas en tres fluidos biológicos simulados, bajo diferentes condiciones de $\mathrm{pH}$ (un rango de 4-9) y variaciones de temperatura. Obteniendo como resultado que la aleación a pH ácido o a altas temperaturas se corroe significativamente [9].

Fekry analizó el comportamiento electroquímico de la aleación Ti6Al4V en una solución de ácido oxálico con el fin de evaluar la influencia de la concentración de iones $\mathrm{Cl}^{-}$y $\mathrm{SO}_{4}{ }^{-2}$ y la temperatura en la velocidad de corrosión. Sus resultados indicaron que el incremento en la concentración de estos iones conduce a un aumento en la velocidad de corrosión [10].

El acero inoxidable 316 LVM ha sido estudiado por su amplia aplicación desde la industria hasta las aplicaciones biomédicas como se mencionó anteriormente. Chun-Che, emplearon técnicas de polarización cíclica para analizar el efecto de diferentes procesos pasivantes sobre la resistencia a la corrosión tanto in vitro como en vivo del acero 316 LVM donde concluyen que las propiedades del óxido formado sobre la superficie en lugar del espesor de este es un factor predominante para explicar la resistencia a la corrosión [11].

Polo y colaboradores realizaron estudios de espectroscopia de impedancia electroquímica sobre el acero $316 \mathrm{LVM}$ en solución de $\mathrm{NaCl} 5 \%$ a temperatura ambiente en las regiones pasivas y de picadura, representando lo obtenido experimentalmente en circuitos equivalentes para su comprensión. Los resultados mostraron un comportamiento capacitivo en la región pasiva y tres blucles capacitivos en la región de picadura y uno de ellos con efecto inductivo [12]. Otros autores han investigado sobre el efecto del $\mathrm{pH}$ en diferentes aceros inoxidables en soluciones de $\mathrm{NaCl}$ [13-14].

En base a lo anterior, la finalidad de este trabajo es evaluar la velocidad de corrosión y la susceptibilidad al picado del acero 316 LVM y la aleación Ti6Al4V ELI en medios con diferentes condiciones de $\mathrm{pH}$ 
y concentración de iones cloruro, mediante el análisis de las siguientes técnicas electroquímicas: Espectroscopia de Impedancia Electroquímica y Polarización Potenciodinámica Cíclica.

\section{MATERIALES Y PREPARACIÓN DE MUESTRAS}

Los materiales empleados fueron Ti6A14V ELI ASTM F-136 y AISI SAE 316L ASTM F-138 con composiciones químicas obtenidas por Espectrometría de chispa son reportadas en las Tablas 1 y 2 que corroboran las composiciones dadas por el proveedor para estos tipos de materiales biocompatibles. Se utilizaron probetas de $12 \mathrm{~mm}$ de diámetro y $2 \mathrm{~mm}$ de espesor, las que fueron desbastadas con papel de lija $\mathrm{N}^{\circ} 240,320,400$ y 600. Luego fueron limpiadas con alcohol etílico para eliminar rastros de grasa.

Tabla 1. Composición química de la aleación Ti6Al4V ELI.

\begin{tabular}{|ll|l|}
\hline \multicolumn{3}{|c|}{ Ti6Al4V ELI ASTM F136 } \\
\hline Aluminio & $(\mathrm{Al})$ & 6,102 \\
\hline Vanadio & $(\mathrm{V})$ & 4,119 \\
\hline Hierro & $(\mathrm{Fe})$ & 0,183 \\
\hline Azufre & $(\mathrm{S})$ & 0,342 \\
\hline Silicio & $(\mathrm{Si})$ & 0,444 \\
\hline Titanio & $(\mathrm{Ti})$ & 88,66 \\
\hline
\end{tabular}

Tabla 2. Composición química del acero inoxidable 316 LVM.

\begin{tabular}{|ll|c|}
\hline \multicolumn{3}{|c|}{ Acero inoxidable 316 LVM } \\
\hline Carbono & $(\mathrm{C})$ & 0,02 \\
\hline Cromo & $(\mathrm{Cr})$ & 18,044 \\
\hline Níquel & $(\mathrm{Ni})$ & 14,012 \\
\hline Manganeso & $(\mathrm{Mn})$ & 1,849 \\
\hline Molibdeno & $(\mathrm{Mo})$ & 2,859 \\
\hline Vanadio & $(\mathrm{V})$ & 0,01 \\
\hline Azufre & $(\mathrm{S})$ & 0,963 \\
\hline Silicio & $(\mathrm{Si})$ & 0,518 \\
\hline Fósforo & $(\mathrm{P})$ & 0,078 \\
\hline
\end{tabular}

La presencia de un alto contenido de azufre, tanto en el acero como en la aleación de titanio, se debe a consideraciones resolutivas de la técnica de medición y posible contaminación.

\section{MONTAJE EXPERIMENTAL}

Las técnicas de Espectroscopia de Impedancia Electroquímica, Resistencia a la Polarización Lineal y Polarización Potenciodinámica Cíclica fueron empleadas para la evaluación, mediante un potenciostato (GAMRY instruments Reference 600TM Potenciostat/galvanostat/ ZRA) y una celda plana vertical de vidrio compuesta por un electrodo de trabajo (probetas de Ti6Al4V o AISI SAE 316L), un electrodo de referencia (calomel) y un contraelectrodo de grafito, cuyo esquema se muestra en la Figura 1.

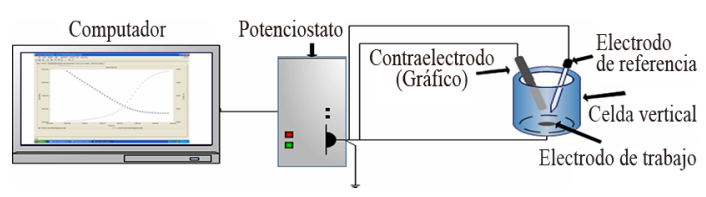

Figura 1. Montaje experimental.

\section{ENSAYOS DE ESPECTROSCOPIA DE IMPEDANCIA ELECTROQUÍMICA (EIS)}

Esta técnica permite la caracterización de las propiedades de materiales, que consiste en la aplicación de una perturbación sinusoidal de potencial eléctrico de frecuencia variable al material estudiado y el registro de la respuesta en corriente dentro de una celda electroquímica. La impedancia se define como el cociente entre el potencial aplicado y la intensidad medida a la salida. En muchos materiales y sistemas electroquímicos la impedancia varía con la frecuencia del potencial aplicado en una forma que está relacionada con las propiedades de dichos materiales. Es uno de los métodos que más información proporciona sobre las propiedades de la interfase biomaterial/ electrolito [13].

Existen dos formas de representar gráficamente los resultados de impedancia obtenidos de un ensayo de EIS: 1) Diagrama de Nyquist, donde se representa la parte imaginaria multiplicada por $-1(-Z$ "), frente a la parte real (Z'). 2)

Diagramas de Bode, donde se representan el logaritmo del módulo de la impedancia $(\log |Z|)$ y el desfase $(\varphi)$ en función del logaritmo de la frecuencia $\log (\omega)$ [14]. 
Para el desarrollo de esta prueba en cada una de las muestras se usó una frecuencia inicial de 50.000 $\mathrm{Hz}$ y una final de $0,1 \mathrm{~Hz}$.

\section{ENSAYOS DE POLARIZACIÓN POTENCIODINÁMICA CÍCLICA}

Este método, descrito en la norma ATM F2129, permite identificar el inicio y propagación de la corrosión localizada, la cual ocurre al potencial en el que la corriente anódica incrementa rápidamente. Al alcanzar dicho límite de corriente se invierte el sentido de la polarización potenciodinámica hasta que la línea de corriente corta la curva de ida formando un ciclo llamado "ciclo de histéresis". Los procesos de picado, una vez iniciados, pueden propagarse a potenciales menos nobles que los de iniciación y el potencial de cierre del ciclo de histéresis suele ser considerado el menor potencial al cual el picado puede aún propagarse, norma ASTM G61. En esta prueba se realizaron barridos potenciodinámicos de $-250 \mathrm{mV}$ y $250 \mathrm{mV}$ con una velocidad de barrido de $1 \mathrm{mV} / \mathrm{s}$.

\section{RESULTADOS Y DISCUSIONES}

Previo a los ensayos realizados en este trabajo, se hizo un diseño y análisis experimental en un programa estadístico para identificar por medio de diagramas de Pareto y de efectos principales, la variable más influyente sobre la velocidad de corrosión. Cada uno de los materiales fue sometido a diferentes condiciones de concentración de $\mathrm{NaCl}(1 \%$ y $3 \%)$ y $\mathrm{pH}(4,5$ y 10$)$. Se obtuvo las velocidades de corrosión instantáneas para cada una de las condiciones experimentales mediante las curvas de Polarización Potenciodinámica Cíclica. Para esto se determinaron las pendientes Tafel por extrapolación de las zonas catódica y anódica de las curvas resultantes, con dichas pendientes se obtuvo la densidad de corriente de corrosión y con la ecuación de Stern-Geary se calculó la resistencia a la polarización. Con estos datos se halló la velocidad de corrosión de acuerdo a la norma ASTM G102.

Ecuación Stern Geary, norma ASTM G3

$$
R_{p}=\frac{b_{a} b_{c}}{2,303\left(b_{a} b_{c}\right) i_{\text {corr }}}
$$

Donde,

$b_{a}=$ Pendiente Tafel anódica

$b_{c}=$ Pendiente Tafel catódica

Ecuación velocidad de corrosión norma ASTM G102.

$$
V_{\text {corr }}=k_{1} \frac{i_{\text {corr }} E W}{\rho} * \frac{1.000}{25,4}
$$

Donde,

$$
\begin{aligned}
& V_{\text {corr }}[=] \mathrm{mpy} \\
& i_{\text {corr }}[=] \mu \mathrm{A} / \mathrm{cm}^{2} \\
& k_{1}=3,27 * 10^{-3} \mathrm{mmg} / \mu \mathrm{Acmy} \\
& \rho=\mathrm{g} / \mathrm{cm}^{3}
\end{aligned}
$$

En la Figura 2 se muestra que los potenciales de corrosión para la solución con concentraciones de $3 \%$ de $\mathrm{NaCl}$ presentan valores más negativos y una tendencia a mayores valores de densidad de corriente con respecto a las soluciones con $1 \%$.

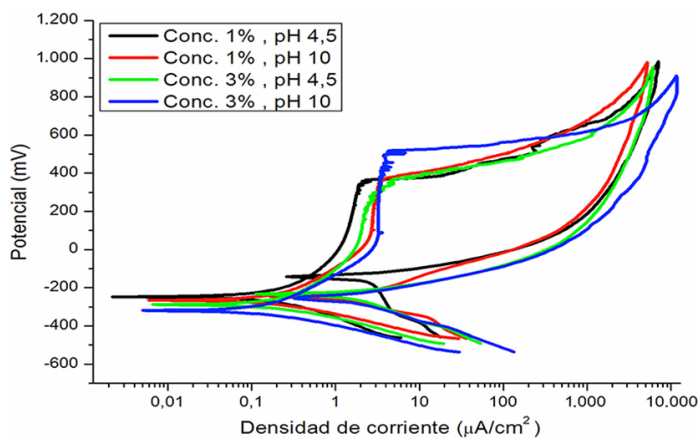

Figura 2. Curvas de Polarización Potenciodinámica Cíclica del acero 316 LVM a diferentes $\mathrm{pH}$.

Por ejemplo, para $\mathrm{pH}$ de 4,5 la velocidad de corrosión fue de 0,1245 mpy con $1 \%$ de $\mathrm{NaCl}$ y de 0,1768 mpy con $3 \%$ de $\mathrm{NaCl}$, presentando además menores valores de velocidad de corrosión en las mismas concentraciones de $\mathrm{NaCl}$ pero a un $\mathrm{pH}$ de 10 (0,0918 mpy y 0,0923 mpy, respectivamente).

En la Figura 3 se muestra el comportamiento de la aleación Ti6A14V bajo las condiciones experimentales establecidas. Se puede observar un desplazamiento de la curva anódica reversa, hacia potenciales más positivos, mostrando el ciclo de histéresis una estrecha correspondencia entre el comportamiento anódico reverso y el directo. 


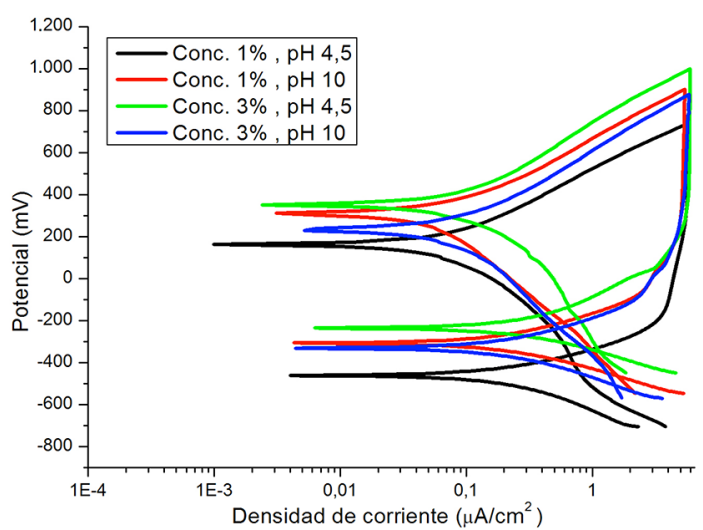

Figura 3. Curvas de Polarización Potenciodinámica Cíclica de la aleación Ti6Al4V ELI a las condiciones experimentales establecidas.

Para $\mathrm{pH}$ de 4,5 la velocidad de corrosión fue de 0,0998 mpy con $1 \%$ de $\mathrm{NaCl}$ y de 0,1014 mpy con $3 \%$ de $\mathrm{NaCl}$, presentando además menores valores de velocidad de corrosión en las mismas concentraciones de $\mathrm{NaCl}$ pero a un $\mathrm{pH}$ de $10(0,0554$ mpy y 0,0728 mpy, respectivamente).

Con el análisis de experimentos basados en estos ensayos preliminares se estableció evaluar el comportamiento del acero inoxidable 316 LVM y la aleación de Ti6Al4V, en un rango amplio de $\mathrm{pH}(4,5-6-7,5-10$ y 12$)$ y a concentración de $3 \%$ de $\mathrm{NaCl}$.

\section{EFECTO DEL PH SOBRE LA VELOCIDAD DE CORROSIÓN DEL ACERO INOXIDABLE 316 LVM}

De acuerdo con el diagrama de Pourbaix propuesto por Yaniv [15] para los aceros inoxidables a $25^{\circ} \mathrm{C}$ en $\mathrm{H}_{2} \mathrm{O}$ (Figura 4), a pH 4,5 y un potencial de corrosión de $-288 \mathrm{mV}$ el material se encuentra en una zona de activación, cuyo producto es el ion $\mathrm{Fe}^{2+}$, por tanto la velocidad de corrosión en este $\mathrm{pH}$ es mayor que en los otros valores de $\mathrm{pH}$ evaluados.

Para los pH 6 - 7,5 - 10 y 12 el acero se encuentra en la zona de pasividad con potenciales de corrosión entre $-252 \mathrm{mV}$ y $-338 \mathrm{mV}$ en los cuales puede haber formación de $\mathrm{Cr}_{2} \mathrm{O}_{3}$ como capa pasiva.

La Figura 5 muestra las curvas de polarización cíclica del acero $316 \mathrm{~L}$ en solución 3\% $\mathrm{NaCl}$ y

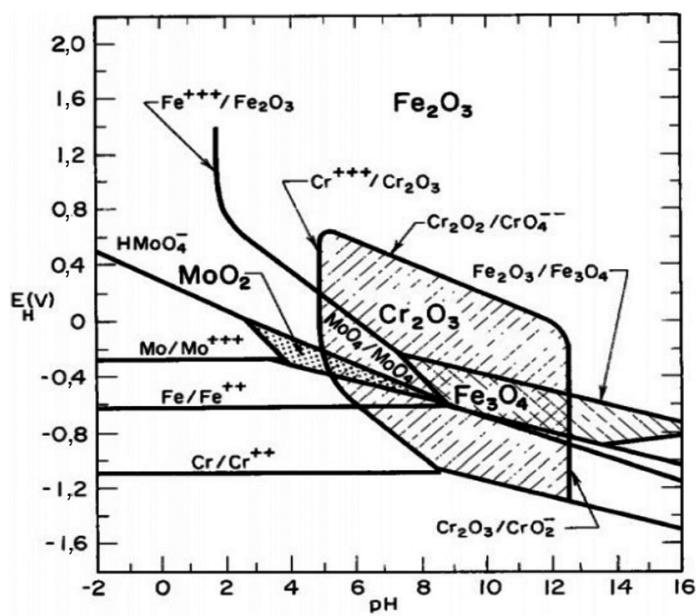

Figura 4. Superposición del diagrama de Pourbaix para el hierro, cromo y molibdeno.

los diferentes valores de $\mathrm{pH}$ evaluados. Se puede observar una zona pasiva más grande en los $\mathrm{pH}$ de 6 - 7,5 y 10 en comparación con el pH de 4,5. En los pH 6 y 7,5 Figura 5b y 5c los valores de potencial de picadura Ep son similares, por tanto en estas condiciones el $\mathrm{pH}$ no influye en el potencial de picadura lo que está de acuerdo a lo reportado por diferentes autores acerca de la corrosión en este tipo de acero inoxidable [16], [17]. En el caso de $\mathrm{pH}$ de 10 hay una leve disminución de Ep, la cual se puede atribuir a un favorecimiento en la cinética de formación de la película pasiva, por la presencia de $\mathrm{OH}^{-}$durante el proceso de polarización anódica [18].

La diferencia entre el potencial de rompimiento Eb y el potencial de picadura Ep, representa la capacidad de repasivación, o la capacidad de impedir la formación de nuevas picaduras, pero permite la evolución de las picaduras ya existentes [16]. Para un $\mathrm{pH}$ de 4,5 esta diferencia presenta un valor pequeño en comparación a los otros valores de $\mathrm{pH}$ evaluados. Esto indica que a valores mayores de $\mathrm{pH}$ el acero inoxidable tiene más capacidad para repasivarse [16, 14]. Por otra parte, la diferencia Eb - Ep no presenta un efecto apreciable con respecto al pH. Sin embargo, se muestra una tendencia de un mayor tamaño de bucle de histéresis al incrementar los valores de $\mathrm{pH}$, a excepción de la gráfica con $\mathrm{pH}$ de 12 , cuya histéresis es similar a la que presenta las aleaciones de titanio, de acuerdo a lo expuesto por Gutiérrez de Cid [19] la ausencia de un bucle 

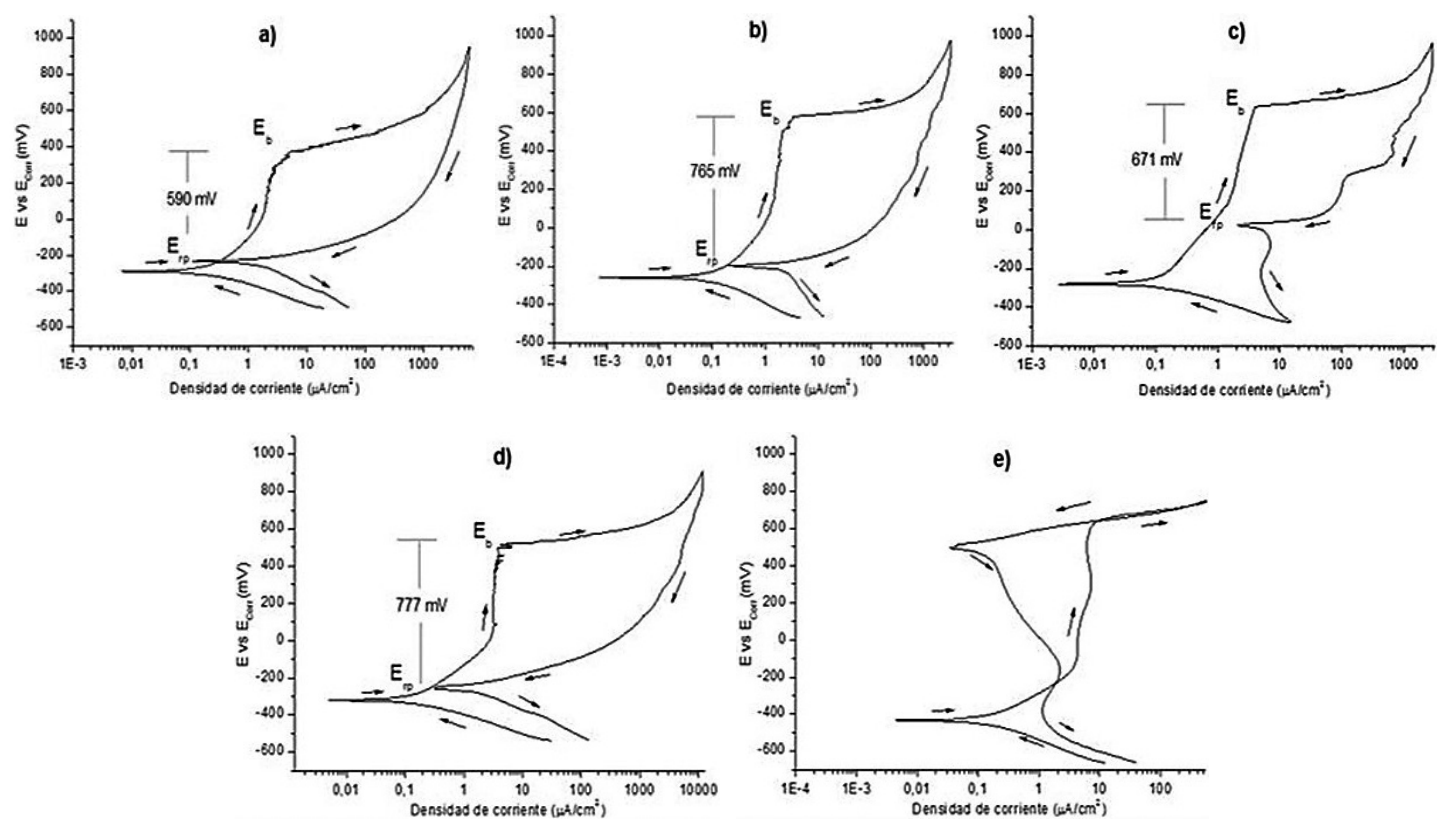

Figura 5. Curvas de Polarización Potenciodinámica Cíclica del acero 316 LVM a condiciones de 3\% de NaCl: a) pH 4,5, b) pH 6, c) pH 7,5, d) pH 10, e) pH 12.

de histéresis indica un fenómeno de corrosión generalizada y se vuelve imposible identificar correctamente los parámetros para evaluar la susceptibilidad a la corrosión localizada [14]. En la Tabla 3 se muestran los resultados de los parámetros de corrosión obtenidos de la técnica de polarización potenciodinámica.

En la Figura 6 se observa la variación de la velocidad de corrosión en el acero 316 LVM con la variación de $\mathrm{pH}$, confirmando claramente un mayor valor a pH de 4,5, una disminución de la corrosión a pH de 6 y un incremento gradual de la velocidad de corrosión hasta $\mathrm{pH}$ de 12.

Tabla 3. Resultados Polarización Potenciodinámica Cíclica para el acero 316 LVM sometido a condiciones de $3 \%$ de $\mathrm{NaCl}$ y diferentes $\mathrm{pH}$.

\begin{tabular}{|c|c|c|c|c|c|}
\hline $\mathbf{p H}$ & $\begin{array}{c}\boldsymbol{\beta A} \\
(\mathbf{V})\end{array}$ & $\begin{array}{c}\boldsymbol{\beta c} \\
(\mathbf{V})\end{array}$ & $\begin{array}{c}\mathbf{I} \text { corr } \\
\left(\mathbf{n A} / \mathbf{c m}^{2}\right)\end{array}$ & $\begin{array}{c}\text { Vcorr } \\
(\mathbf{m p y})\end{array}$ & $\begin{array}{c}\mathbf{R p} \\
\left(\mathbf{k} \mathbf{\mathbf { s }}-\mathbf{c m}^{2}\right)\end{array}$ \\
\hline 4,5 & 0,258 & 0,1214 & 365,9 & 0,1768 & 79,29 \\
\hline 6 & 0,285 & 0,1478 & 113,0 & 0,0546 & 35,80 \\
\hline 7,5 & 0,387 & 0,1116 & 157,0 & 0,0764 & 159,70 \\
\hline 10 & 0,194 & 0,1100 & 191,0 & 0,0923 & 111,10 \\
\hline 12 & 0,287 & 0,1257 & 208,3 & 0,1011 & 125,40 \\
\hline
\end{tabular}

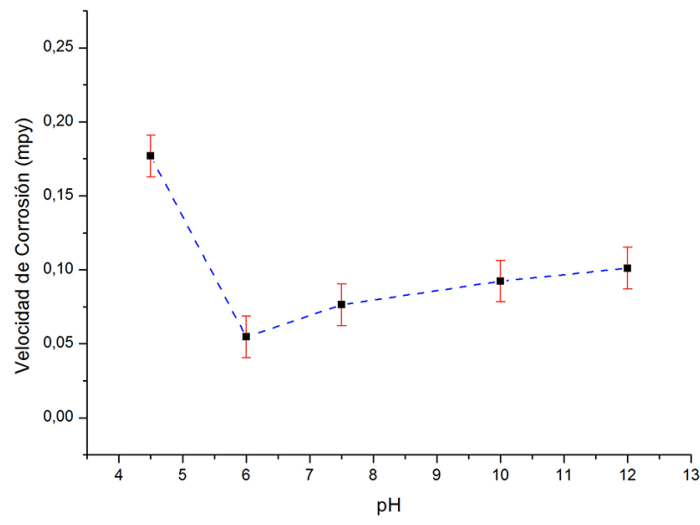

Figura 6. Velocidad de corrosión del acero 316 LVM a condiciones de $3 \%$ de concentración de $\mathrm{NaCl}$ y diferentes $\mathrm{pH}$.

Este incremento de la velocidad de corrosión hasta $\mathrm{pH}$ de 12 se puede atribuir a la posible presencia de productos de magnetita, de acuerdo a la correspondencia con el diagrama de Pourbaix de la Figura 4, el que es un compuesto inestable que genera apreciables incrementos en la velocidad de corrosión.

Las mediciones de Espectroscopia de Impedancia Electroquímica se muestran en un diagrama Nyquist 
(Figura 7). El análisis se realiza a partir de un circuito equivalente que se ajusta a los resultados obtenidos (Figura 8). Un circuito equivalente con dos constantes de tiempo describe el mecanismo de corrosión en las interfaces solución/óxido y óxido/metal [16].

Los elementos que conforman el circuito son $R_{s}$ resistencia a la solución, $R_{c t}$ resistencia a la transferencia de carga, $C P E_{d l}$ es la capacitancia de la múltiples capas de productos de corrosión incluyendo los defectos sobre ella, incluidos la picadura, $C P E_{p e l}$ la capacitancia de la capa pasiva y $R_{p e l}$ la resistencia de la película [16].

Los parámetros eléctricos se obtuvieron mediante el software Zview en el que se evaluó la calidad del ajuste del modelo con valores de chi cuadrado menores a $10^{-3}$. Estos se encuentran reportados en la Tabla 4.

Inicialmente se aprecia que a frecuencias altas las resistencias a la solución $R_{S}$ son similares tal como se aprecia en el diagrama de Nyquist de la Figura 7.

Por otra parte, los valores de la $R_{p e l}$ son mucho más grandes que los valores de la $R_{c t}$, según algunos autores [20] esto se explica por la protección que genera la capa pasiva. Se observa en la solución con pH 7,5 que $R_{p e l}$ toma su valor máximo mientras que a $\mathrm{pH} 4,5$ y 12 los valores son más bajos.

Se realizó una micrografía (Microscopio Nikon Eclipse Lv1000) sobre la probeta de acero 316 LVM en condiciones de $\mathrm{pH} 10 \mathrm{y}$ concentraciones del $3 \%$

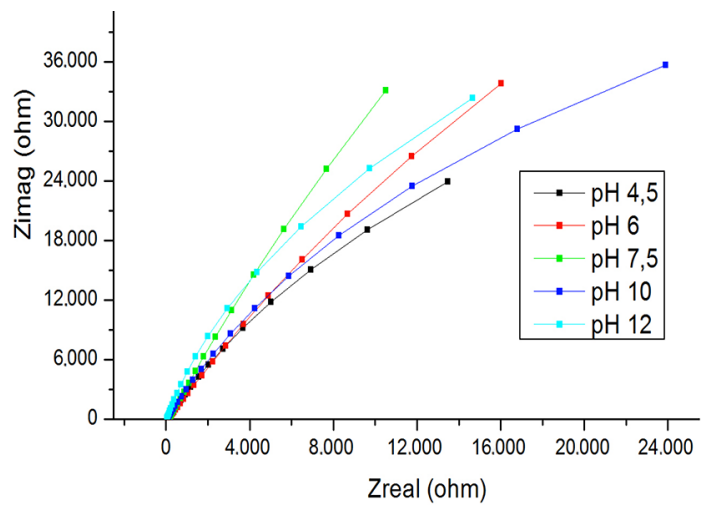

Figura 7. Diagrama de Nyquist para el acero 316LVM a condiciones de concentración de $3 \%$ de $\mathrm{NaCl}$ y diferentes $\mathrm{pH}$.

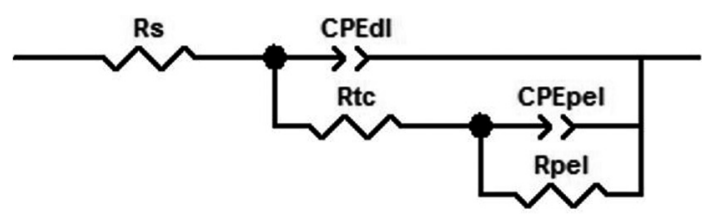

Figura 8. Circuito equivalente propuesto.

para la observación de picaduras representativas de aproximadamente 400-500 $\mu \mathrm{m}$ (Figura 9).

La Figura 9d representa el modelo 3D de la picadura a) con una profundidad de alrededor de $100 \mu \mathrm{m}$. $\mathrm{La}$ morfología de picado observada en estas micrografías, permite establecer una forma de picado estable, en donde solo se presenta rompimiento de la capa de óxido y repasivación, no se aprecia un importante daño a nivel microestructural en la aleación de acero base.

Tabla 4. Valores de las resistencias y capacitancias que conforman el circuito equivalente simulado en el software Zview para el acero 316 LVM a condiciones de $3 \%$ de concentración de $\mathrm{NaCl}$ y diferentes $\mathrm{pH}$.

\begin{tabular}{|c|c|c|c|c|c|c|c|c|}
\hline \multirow[b]{2}{*}{ pH } & \multirow{2}{*}{$\begin{array}{c}R_{s} \\
\left(\Omega-c m^{2}\right)\end{array}$} & \multicolumn{2}{|c|}{$C P E_{d l}$} & \multirow{2}{*}{$\begin{array}{c}\boldsymbol{R}_{t c} \\
\left(\boldsymbol{\Omega}-\mathrm{cm}^{2}\right)\end{array}$} & \multicolumn{2}{|c|}{$C P E_{p e l}$} & \multirow{2}{*}{$\begin{array}{c}\boldsymbol{R}_{p e l} \\
\left(\mathbf{k} \boldsymbol{\Omega}-\mathrm{cm}^{2}\right)\end{array}$} & \multirow{2}{*}{$\begin{array}{c}\text { Chi } \\
\text { cuadrado }\end{array}$} \\
\hline & & $\begin{array}{l}C P E_{d l} T \\
\left(\mu \mathrm{F} / \mathrm{cm}^{2}\right)\end{array}$ & $\begin{array}{c}C P E_{d l}-P \\
(-)\end{array}$ & & $\begin{array}{l}C P E_{p e l} T \\
\left(\mu \mathrm{F} / \mathrm{cm}^{2}\right)\end{array}$ & $\begin{array}{c}C P E_{2}-P \\
(-)\end{array}$ & & \\
\hline 4,5 & 27,5 & 5,5 & 0,64 & 77,92 & 4,60 & 0,82 & 123,5 & 0,0003 \\
\hline 6 & 27,31 & 11,5 & 0,71 & 143,2 & 28,10 & 0,81 & 508,8 & 0,0004 \\
\hline 7,5 & 50,73 & 14,7 & 0,86 & 225,9 & 29,30 & 0,83 & 791,8 & 0,0002 \\
\hline 10 & 27,79 & 7,1 & 0,81 & 12,66 & 47,00 & 0,83 & 137,46 & 0,00004 \\
\hline 12 & 29,76 & 26,8 & 0,91 & 31,59 & 14,04 & 0,87 & 133,09 & 0,00005 \\
\hline
\end{tabular}




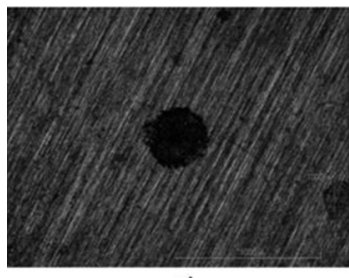

a)

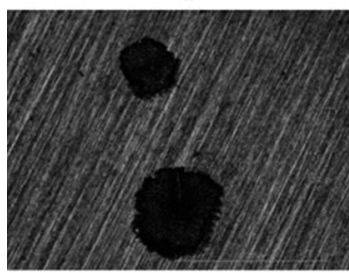

c)

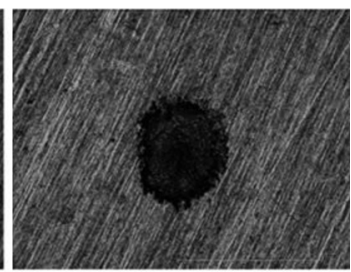

b)

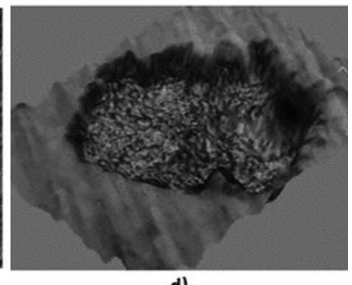

d)
Figura 9. Micrografía de acero 316 LVM. a) Picadura de $400 \mu \mathrm{m}$ de diámetro

b) Picadura de $600 \mu \mathrm{m}$ de diámetro

c) Picadura de 300 y $500 \mu \mathrm{m}$ de diámetro d) Modelo 3D de a).

\section{EFECTO DEL PH SOBRE LA VELOCIDAD DE CORROSIÓN DE LA ALEACIÓN Ti6Al4V}

En las curvas de Polarización Potenciodinámica Cíclica de la Figura 10 se observa que en ninguno de los casos se presentó corrosión localizada, lo que es

un comportamiento característico de esta aleación. También se puede apreciar que la tendencia a la pasivación, $\left|E_{c o r r}-E_{r p}\right|$ presenta valores más bajos a pH mayor de 4,5 lo que indica una menor ventana de pasivación a pH más ácido. En la Tabla 5, los parámetros de $i_{\text {corr }}$ y $V_{\text {corr }}$ a valores de $\mathrm{pH}$ alcalino, son menores comparados con los de $\mathrm{pH}$ ácido, lo cual confirma la existencia de una capa pasiva más estable a estas condiciones.

El valor de la pendiente anódica $\mathrm{B}_{\mathrm{A}}$ es mayor comparado con el valor de la pendiente catódica $\mathrm{B}_{\mathrm{C}}$ a $\mathrm{pH}$ alcalino, lo que puede asociarse a la presencia de un control anódico en el proceso de corrosión y la formación de una capa pasiva.

Tabla 5. Resultados de Polarización Potenciodinámica Cíclica para el Ti6Al4V a condiciones de concentración de $3 \%$ de $\mathrm{NaCl}$ y diferentes $\mathrm{pH}$.

\begin{tabular}{|c|c|c|c|c|r|}
\hline $\mathbf{p H}$ & $\begin{array}{c}\boldsymbol{\beta A} \\
(\mathbf{V})\end{array}$ & $\begin{array}{c}\boldsymbol{\beta c} \\
(\mathbf{V})\end{array}$ & $\begin{array}{c}\mathbf{I} \text { corr } \\
\left(\mathbf{n A} / \mathbf{c m}^{2}\right)\end{array}$ & $\begin{array}{c}\text { Vcorr } \\
(\mathbf{m p y})\end{array}$ & $\begin{array}{c}\mathbf{R p} \\
\left(\mathbf{k} \mathbf{\Omega}-\mathbf{c m}^{2}\right)\end{array}$ \\
\hline 4,5 & 0,1835 & 0,2311 & 249,6 & 0,0998 & 177,94 \\
\hline 6 & 0,2018 & 0,2113 & 187,4 & 0,0749 & 239,23 \\
\hline 7,5 & 0,2793 & 0,2671 & 55,4 & 0,0222 & $1.069,88$ \\
\hline 10 & 0,1594 & 0,1403 & 38,1 & 0,0152 & 849,87 \\
\hline 12 & 0,1755 & 0,1542 & 27,0 & 0,0108 & $1.321,87$ \\
\hline
\end{tabular}

b)
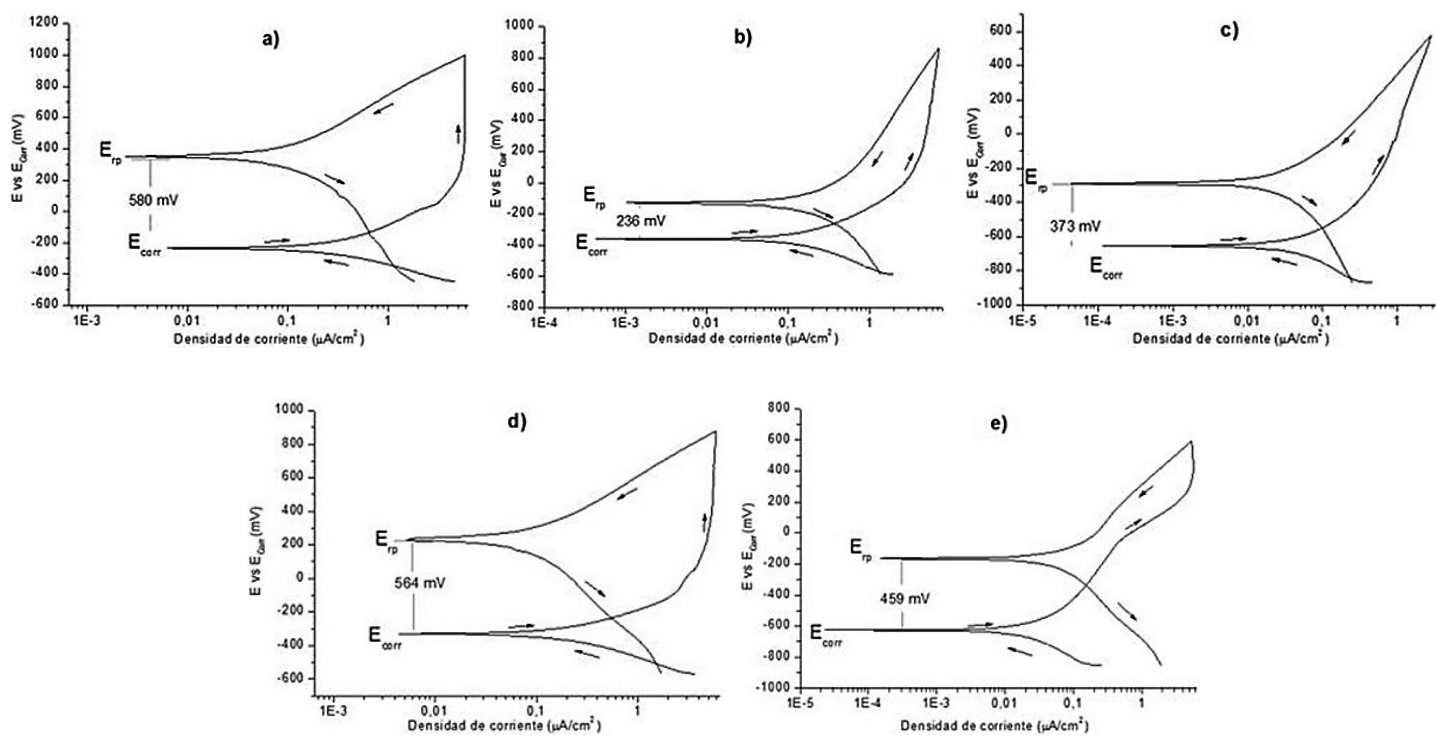

Figura 10. Curvas de Polarización Potenciodinámica Cíclica del Ti6Al4V, concentración 3\% de $\mathrm{NaCl}$ : a) $\mathrm{pH} 4,5, \mathrm{~b}) \mathrm{pH} 6, \mathrm{c}) \mathrm{pH} 7,5$, d) $\mathrm{pH} 10$, e) $\mathrm{pH} 12$. 
En la Figura 11 se observa la variación de la velocidad de corrosión en la aleación Ti6A14V ELI a diferentes condiciones de $\mathrm{pH}$.

Al igual que el acero 316 LVM se observa un mayor valor de corrosión a $\mathrm{pH}$ de 4,5 , pero una disminución gradual de la velocidad de corrosión hasta $\mathrm{pH}$ de 12.

En las pruebas de Espectroscopia de Impedancia Electroquímica del Ti6Al4V ELI se obtuvo, en el diagrama de Nyquist, curvas que se asemejan a semicírculos incompletos Figura 12. Se ve claramente una amplitud mayor en las curvas que representan condiciones de $\mathrm{pH}$ alcalino, lo que describe un mejor comportamiento capacitivo, una capa protectora pasiva mejor.

Aunque la naturaleza de la capa pasiva entre el cero y la aleación de titanio analizado difieren, los espectros

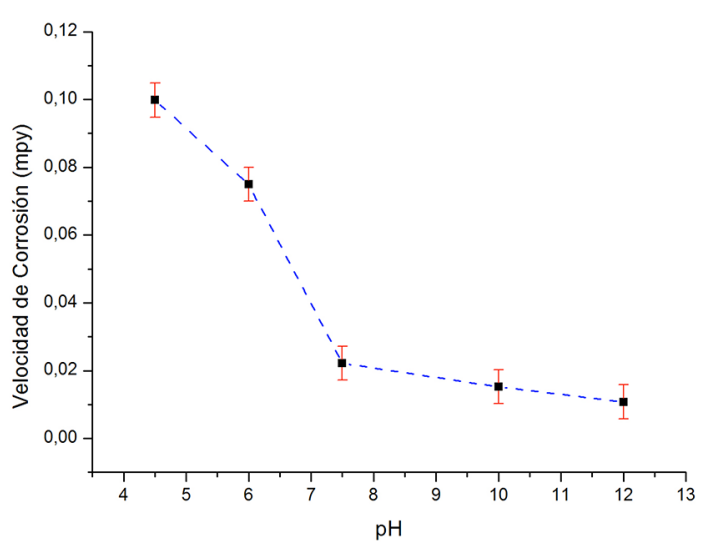

Figura 11. Velocidad de corrosión del Ti6Al4V ELI a condiciones de $3 \%$ de concentración de $\mathrm{NaCl}$ y diferentes $\mathrm{pH}$.

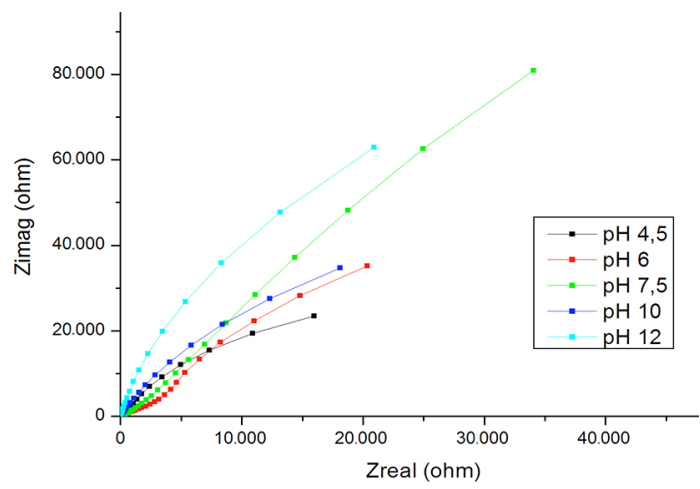

Figura 12. Diagrama de Nyquist para el Ti6Al4V ELI a condiciones de concentración de $3 \%$ de $\mathrm{NaCl}$ y diferentes $\mathrm{pH}$.

obtenidos de la aleación de titanio se ajustaron a un circuito equivalente similar al obtenido en el acero 316 LVM; sin embargo, los procesos de corrosión son descritos de forma diferente de acuerdo con los parámetros eléctricos obtenidos. En la Tabla 6 se muestran los resultados obtenidos a partir del ajuste al modelo de circuito equivalente propuesto.

Se muestra que todos los casos estudiados tienen un valor de $\mathrm{R}_{\mathrm{pel}}$ mayor al de $\mathrm{R}_{\mathrm{tc}}$ indicando que la interface óxido-metal tiene una alta resistencia a la transferencia de carga (Tabla 6).

Las técnicas de polarización potenciodinámica y de espectroscopia de impedancia electroquímica han permitido establecer criterios basados en corrosión del acero inoxidable 316 LVM y la aleación Ti6Al4V, empleados en la fabricación de prótesis óseas, haciendo énfasis en los cambios de $\mathrm{pH}$ que se presentan en los ambientes fisiológicos en las diferentes etapas de osteogénesis y osteointegración.

Tabla 6. Valores de las resistencias y capacitancias que conforman el circuito equivalente simulado en el software Zview para el Ti6Al4V a condiciones de 3\% de concentración de $\mathrm{NaCl}$ y diferentes $\mathrm{pH}$.

\begin{tabular}{|c|c|c|c|c|c|c|c|c|}
\hline \multirow[b]{2}{*}{ pH } & \multirow{2}{*}{$\begin{array}{c}\boldsymbol{R}_{s} \\
\left(\Omega-\mathrm{cm}^{2}\right)\end{array}$} & \multicolumn{2}{|c|}{$C P E_{d l}$} & \multirow{2}{*}{$\begin{array}{c}\boldsymbol{R}_{t c} \\
\left(\Omega-\mathrm{cm}^{2}\right)\end{array}$} & \multicolumn{2}{|c|}{$C P E_{p e l}$} & \multirow{2}{*}{$\begin{array}{c}\boldsymbol{R}_{p e l} \\
\left(k \Omega-c m^{2}\right)\end{array}$} & \multirow{2}{*}{$\begin{array}{c}\text { Chi } \\
\text { cuadrado }\end{array}$} \\
\hline & & $\begin{array}{c}C P E_{d l} T \\
\left(\mu F / \mathrm{cm}^{2}\right)\end{array}$ & $\begin{array}{c}C P E_{d l} P \\
(-)\end{array}$ & & $\begin{array}{l}C P E_{p e l} T \\
\left(\mu F / c m^{2}\right)\end{array}$ & $\begin{array}{c}C P E_{2^{-}} P \\
(-)\end{array}$ & & \\
\hline 4,5 & 32,86 & 2,80 & 0,65 & 448,0 & 44,90 & 0,91 & 66,59 & 0,00004 \\
\hline 6 & 27,01 & 28,2 & 0,63 & $11.925,0$ & 8,49 & 1,00 & 2.638 & 0,0004 \\
\hline 7,5 & 186,3 & 9,05 & 0,81 & $3.910,0$ & 8,04 & 0,78 & $1.607,6$ & 0,0004 \\
\hline 10 & 26,64 & 11,50 & 0,85 & 24,77 & 24,60 & 0,87 & 145,30 & 0,0001 \\
\hline 12 & 26,03 & 18,10 & 0,93 & 41,33 & 4,79 & 0,95 & 295,51 & 0,00003 \\
\hline
\end{tabular}


De acuerdo con la norma ASTM F2129, la aceptación de biomateriales de naturaleza metálica está basada en la capacidad del material de formar una capa protectora estable, de resistir la picadura producida por especies agresivas y la facilidad de recuperarse ante el daño localizado. En ese orden de ideas el análisis de corrosión de estos materiales sometidos a estudio basado en la variación de $\mathrm{pH}$, permite identificar el comportamiento de los materiales ante situaciones agresivas del medio fisiológico, que se pueden extrapolar a situaciones in vivo. Los resultados y análisis determinaron que el tamaño del bucle de histéresis en los ensayos potenciodinámicos es un adecuado criterio de aceptación y de control de los procesos de integración del biomaterial metálico con el hueso y el ambiente fisiológico que lo rodea. Sin embargo, ambos materiales tratados en este trabajo deben cumplir con requisitos diferentes de aceptación de acuerdo con los parámetros obtenidos de las curvas potenciodinámicas y de la naturaleza de sus óxidos protectores correspondientes. Por ejemplo, en el caso del acero $316 \mathrm{LVM}$, la norma ASTM F2129 se refiere específicamente al criterio correspondiente a la diferencia entre el potencial de picadura y el potencial de corrosión (a mayor sea esta diferencia, más aceptación). Diferente criterio es aplicable a los dispositivos para uso óseo fabricados en Ti6Al4V ELI, ya que este parece no ser muy claro; sin embargo, de acuerdo a los resultados obtenidos en este trabajo se puede proponer que la mejor aceptación correspondería a la condición que presenta una mayor histéresis en el barrido potenciodinámico, tanto positivo como de vuelta en potencial. En otras palabras, se refiere a la mejor capacidad del material de reparar el daño por corrosión localizada y de mantener una alta estabilidad respecto a la agresividad del ambiente fisiológico.

Paralelamente, en la variación de $\mathrm{pH}$ con la estabilidad del óxido protector en ambos materiales $\left(\mathrm{Cr}_{2} \mathrm{O}_{3}, \mathrm{TiO}_{2}\right)$, la posible presencia de productos de corrosión que son inestables a determinados $\mathrm{pH}$. Igualmente, otros factores como la rugosidad, formas geométricas, procesos de pasivación y especies en la solución fisiológica como sales y proteínas, también pueden generar variaciones en los potenciales de corrosión, que los ubicarían en zonas de inestabilidad, refiriéndose a los diagramas de Pourbaix con lo que los criterios de aceptación podrían ser evaluados para cada caso en especial.

\section{CONCLUSIONES}

En los casos estudiados, se evidencia en el ciclo de histéresis del Ti6Al4V ELI, la ausencia de aumento brusco en la corriente, lo que indica la alta resistencia de este material a la corrosión localizada. Por el contario, para el acero $316 \mathrm{LVM}$ el ciclo de histéresis presenta un aumento significativo en la corriente, señalando que este material es más susceptible a la formación de picaduras.

La velocidad instantánea de corrosión de ambos materiales es mayor a condiciones de $\mathrm{pH} 4,5$ y concentraciones de $3 \%$ de $\mathrm{NaCl}$. Es importante resaltar que la velocidad para el acero 316 LVM es superior a la del Ti6Al4V en todos los casos.

De acuerdo con la norma ASTM F2129 el acero 316 LVM presentó más aceptación a una mayor diferencia entre el potencial de picadura y el potencial de corrosión. En el caso del Ti6Al4V ELI, la mejor aceptación correspondería a la condición que presenta una mayor histéresis en el barrido potenciodinámico. Sin embargo, como caso particular y contrario al acero 316LVM, en las aleaciones de titanio la dirección de histéresis en el sentido contrario de las manecillas del reloj, no está relacionada con corrosión localizada, donde los factores de $\mathrm{pH}$, disminuye en casos específicos la histéresis la que está relacionada con la formación sinérgica de compuestos hidroxilados y óxidos de titanio.

\section{AGRADECIMIENTOS}

Los autores agradecen el apoyo del Sistema General de Regalías de Colombia de (SGR) Macroproyecto de Salud BPIN, código: 2012000100172 por financiar esta investigación.

\section{REFERENCIAS}

[1] C. Elias, J. Lima, R. Valiev and M. Meyers. "Biomedical Applications of Titanium and its Alloys". Biological Materials Science, pp. 46-49. 2008.

[2] W. Chrzanowski, J. Szewczenko, J. TyrlikHeld, J. Marciniak and J. Zak. "Influence of the anodic oxidation on the physico chemical properties of the Ti6A14V ELI alloy". Journal of Materials Processing Technology. Vol. 162-163, pp. 163-168. 2005. 
[3] F.J. Gil, M.P. Ginebra y J. A. Planell. "Metales y aleaciones para la substitución de tejidos". Biomecánica. Vol. 7 № 13, pp. 73-78. 1999.

[4] B. Pérez Rojo. "Biomateriales: Aplicación a cirugía ortopédica y traumatológica". Madrid, España. 2010.

[5] D. Durgalakshmi and R. Ajay Rakkesh. "Stacked Bioglass/TiO2nanocoatings on titanium substrate forenhanced osseointegration and its electrochemical corrosion studies". Applied Surface Science. Vol. 349, pp. 561569. 2015.

[6] ASTM Standard F2129-08, 2008. "Standard Test Method for Conducting Cyclic Potentiodynamic Polarization Measurements to Determine the Corrosion Susceptibility of Small Implant Devices". ASTM International. West Conshohocken, PA, USA. 2003. DOI: 10.1520/F2129-08. URL: www.astm.org.

[7] I. Cvijović-Alagic, Z. Cvijović, J. Bajat and M. Rakin. "Composition and processing effects on the electrochemical characteristics of biomedical titanium alloys". Corrosion Science. Vol. 83, pp. 245-254. 2014.

[8] R. Wen-Wei Hsu, C.C. Yang, C.A. Huang andY.S. Chen. "Investigation on the corrosion behavior of Ti-6Al-4V implant alloy by electrochemical techniques". Materials Chemistry and Physics. Vol. 86, pp. 269-278. 2004.

[9] A.M. Fekry. "The influence of chloride and sulphate ions on the corrosion behavior of $\mathrm{Ti}$ and Ti-6Al-4V alloy in oxalic acid". Electrochimica Acta. Vol. 54, pp. 3480-3489. 2009.

[10] S. Chun-Che, S. Chun-Ming, S. Yea-Yang, J.S. Lin Hui, C. Mau-Song and L. ShingJong. "Effect of surface oxide properties on corrosión resistance of $316 \mathrm{~L}$ stainless steel for biomedical applications". Corrosion Science. Vol. 46 No 2, pp. 427-441. 2004.

[11] J.L. Polo, C.L. Torres, E. Cano y J. M. Bastidas. "Estudio de impedancia de la corrosión del acero inoxidable AISI 316L en las regiones pasiva y de picadura". Metalurgia. Vol. 35, pp. 368-378. 1999.

[12] C.T. Liu and J.K. Wu. "Influence of pH on the passivation behavior of 254SMO stainless steel in $3.5 \% \mathrm{NaCl}$ solution". Corrosion Science. Vol. 49, pp. 2198-2209. 2007.
[13] M.J. Carmezim, A.M. Simoes, M.F. Montemor and M. Da Cunha Belo. "Capacitance behaviour of passive films on ferritic and austenitic stainless steel". Corrosion Science. Vol. 47, pp. 581-591. 2005.

[14] C. Escriva Cerdán. "Caracterización de la pasividad del acero inoxidable UNS N08031 en ácido fosfórico contaminado mediante técnicas electroquímicas". Valencia, España. 2013.

[15] A.E. Yaniv, J.B. Lumsden and R.W. Staehle. "The Composition of Passive films on Ferritic Stainless Steels". Journal Electrochemical. Vol. $124 \mathrm{~N}^{\circ}$ 4, pp. 490-496. 1977.

[16] V. Guiñón-Pina, A. Igual-Muñoz and J. García-Antón. "Influence of $\mathrm{pH}$ on the electrochemical behavior of a duplex stainless steel in highly concentrated $\mathrm{LiBr}$ solutions". Corrosion Science. Vol. 53, pp. 575-581. 2011.

[17] R. Ovarfort. "Critical pitting temperature measurements of stainless steels with an improved electrochemical method". Corrosion Science. Vol. 29, pp. 987-993. 1989.

[18] A.U. Malik, P.C. Mayan Kutty, N.A. Siddiqi, I.N. Andijani and S. Ahmed. "The influence of $\mathrm{pH}$ and chloride concentration on the corrosión behaviour of AISI 316L steel in aqueous solutions". Corrosion Science. Vol. 33, p. 1809-1827. 1992.

[19] A. Pardo Gutiérrez del Cid, E. Otero Huerta, M. C. Merino Casals y M.D. López González. "Estudio de la resistencia a la corrosión localizada del acero inoxidable superaustenítico $24,1 \mathrm{Cr} 22 \mathrm{Ni} 7,1 \mathrm{Mo}$ en mezclas que contienen iones cloruro y cloruro-fluoruro". Revista de Metalurgia Madrid. Vol. 37, pp. 499-508. 2001.

[20] J. Pan, C. Leygraf, R.F.A. JargeliusPettersson and J. Linden. "Characterization of high-temperature oxide films on stainless steels by electrochemical-impedance spectroscopy". Oxidation of Metals. Vol. 50, pp. 431-455. 1998.

[21] Y. Wu, N. Rui-Cheng and H. Hui-Zhong, "The behavior of chromium and molybdenum in the propagation process of localized corrosion of steels”. Corrosion Science. Vol. $24 \mathrm{~N}^{\mathrm{o}} 8$, pp. 691-707. 1984. 